\title{
Visual Cortical Plasticity Following Unilateral Sensorimotor Cortical Lesions in the Neonatal Rat
}

\author{
Eleftherios Stelios Papathanasiou
}

Loyola University Chicago

Follow this and additional works at: https://ecommons.luc.edu/luc_theses

Part of the Neuroscience and Neurobiology Commons

\section{Recommended Citation}

Papathanasiou, Eleftherios Stelios, "Visual Cortical Plasticity Following Unilateral Sensorimotor Cortical Lesions in the Neonatal Rat" (1996). Master's Theses. 4033.

https://ecommons.luc.edu/luc_theses/4033

This Thesis is brought to you for free and open access by the Theses and Dissertations at Loyola eCommons. It has been accepted for inclusion in Master's Theses by an authorized administrator of Loyola eCommons. For more information, please contact ecommons@luc.edu. (c) $($ ) $\Theta \Theta$

This work is licensed under a Creative Commons Attribution-Noncommercial-No Derivative Works 3.0 License. Copyright (c) 1996 Eleftherios Stelios Papathanasiou 
LOYOLA UNIVERSITY CHICAGO

\title{
VISUAL CORTICAL PLASTICITY FOLLOWING UNILATERAL \\ SENSORIMOTOR CORTICAL LESIONS IN THE NEONATAL RAT
}

\author{
A THESIS SUBMITTED TO \\ THE FACULTY OF THE GRADUATE SCHOOL \\ IN CANDIDACY FOR THE DEGREE OF \\ MASTER OF SCIENCE
}

DEPARTMENT OF CELL BIOLOGY, NEUROBIOLOGY \& ANATOMY

BY

ELEFTHERIOS STELIOS PAPATHANASIOU

CHICAGO, ILLINOIS

JANUARY 1996 
Copyright by Eleftherios Stelios Papathanasiou, 1996 All rights reserved. 


\section{ACKNOWLEDGEMENTS}

I wish to thank Dr. Wendy Tillotson for allowing me to work in this field. I would also like to thank Dr. Anthony Castro and Dr. Edward Neafsey for their advice, and Dr. Neal Peachey and Dr Yoshinobu Goto for their help in creating our visual evoked potential protocol. My thanks to Tom Hogan for his excellent technical assistance, and Jennifer Jones, Laia Acarin and Diane Stancik for their help and support. I would also like to thank Linda Fox and Shih-Yen Tsai for their expert assistance in carrying out the photography. This work was supported by VA merit award and Banes Charitable Trust Fund. 
Chapter

1. INTRODUCTION.

Purpose

Review of the Literature

Neuroplasticity: What is it and what is its Purpose?

Normal Brain Development of the Neonatal Rat

Effects of Unilateral Frontal Cortical Lesions on the Structure of the Unablated Hemisphere

Effects of Unilateral Frontal Cortical Lesions on the Structure of the Cerebral Cortex

Effects of Unilateral Frontal Cortical Lesions on Cortical Efferents in the Unablated Hemisphere

Effects of Unilateral Frontal Cortical Lesions on Cortical Afferents in the Unablated Hemisphere

Effects of Lesions on Cerebral Cortical Topography Intramodal Effects of Lesions in the Neonate 
Intramodal Effects of Lesions in the Adult

Crossmodal Effects of Lesions

Review of Methodology of Visual Evoked Potentials (VEPs) in Rats

Review of the Possible Mechanisms of General Cortical Plasticity

2. MATERIALS AND METHODS

Animals

Neonatal Surgery

Flash Visual Evoked Potentials (VEPS)

Intracortical Microstimulation

Tissue Collection and Preparation

Data Analysis

3. RESULTS

Electrophysiological Analysis of Control Rats

Analysis of the SMC Lesions

Electrophysiological Analysis of Rats with SMC Lesions

Analysis of the Electrophysiological Studies from OC Lesioned Rats

Histology of the Visual and Motor Maps in Normal and SMC Lesioned Rats 
4. DISCUSSION AND CONCLUSION. 21

Summary of the Results

A Comparison of our Results to Past Findings

Possible Mechanisms of Neuroplasticity

Cross-modal Neuroplasticity

Plasticity of the Optic Pathway

Future Work

Conclusion

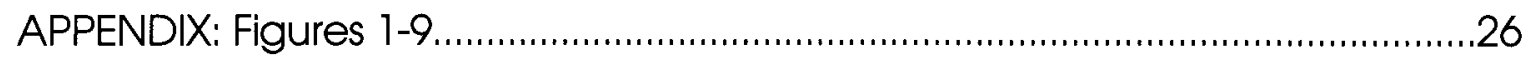

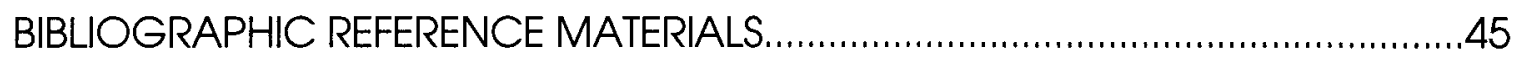

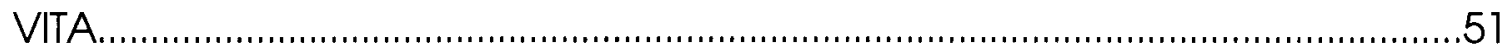




\section{LIST OF ILLUSTRATIONS}

Figure

Page

1. A Comparison of VEPs Obtained at Different Points on One Hemisphere Using Contralateral Eye Stimulation

2. Visual and Motor Maps of Control and SMC Lesioned Rats.

3. Gross and Microscopical Analyses of SMC Lesioned Brains.

4. The Outlines of Unilateral SMC Lesions of Seven Rats and

Unilateral OC Lesions of Six Rats are Shown Superimposed

5. Regression Plots for Normal and SMC Lesioned Rats where the Most Rostral Point of the Visual Map using Ipsilateral Eye Stimulation is Plotted Against the Results Obtained using Contralateral Eye Stimulation.

6. Representation of the Visual Maps of Mature Rats that had Sustained Unilateral $O C$ Lesions as Neonates.

7. Diagrammatic Representations of Coronal Sections of the $O C$ Lesioned Brain Represented by $\S$ in Figure 6, Showing the Extent and Depth of the Lesion.

8. Sagittal Sections of a Hemisphere from a Normal Rat and the Unlesioned Hemisphere from an SMC Rat Stained with Toluidine Blue and at $3.0 \mathrm{~mm}$ Lateral to the Midline.

9. Diagrammatic Representations of Coronal Sections of a Rat Brain Taken Either at the Level of the Sensorimotor or Visual Cortex, and Showing a Corpus Callosal Fibre Running Elther from the Ablated to the Unablated Hemisphere or from the Unablated to the Ablated Hemisphere. 


\section{LIST OF TABLES}

Table

Page

1. Comparison of VEP Results from Normal Rats using Contralateral and Ipsilateral Eye Stimulation with respect to the hemisphere recorded.

2. Summary of the statistical analyses comparing the rostral borders of the visual maps of lesioned rats and their maxima to controls using contralateral eye stimulation

3. Summary of the statistical analyses comparing the rostral borders of the visual maps of lesioned rats and their maxima to controls using ipsilateral eye stimulation 
LIST OF ABBREVIATIONS

bic Bicuculine Methobromide

CNS Central Nervous System

CST Corticospinal Tract

EEG Electroencephalogram

GABA Gamma Amino Butyric Acid

OC Occipital Cortical

SMC Sensorimotor Cortical

VEP Visual Evoked Potential 


\section{CHAPTER 1}

\section{INTRODUCTION}

\section{Purpose}

The purpose of this research is to study the effects of neonatal unilateral sensorimotor cortical lesions on the cortical development of Long-Evans, black-hooded rats. Such cortical lesions are known to cause changes in motor corticofugal pathways from the unablated hemisphere, including the formation of a bilateral corticospinal tract (5) and bilateral projections to the red nucleus, superior colliculus and pons $(7,8,22,30,35)$. Furthermore, microstimulation studies of the unablated hemisphere have shown that the motor representation for forelimb and hindlimb cortex relocates to a more rostral position (51). Although many studies have examined the effect of sensorimotor cortical lesions in the opposite unablated sensorimotor cortex, very little is known about the effect of this lesion on other sensory modalities in the unablated hemisphere. Crossmodal effects of unilateral lesions have so far only been seen in the hemisphere directly affected by the lesion.

The electrophysiological data reported here support the hypothesis that unilateral sensorimotor cortical lesions in the neonatal rat cause a rostral movement not only of the motor but also of the visual map in the unablated hemisphere in the adult. 


\section{Review of the Literature}

Neuroplasticity: What is it and What is its Purpose?

Neuroplasticity has been defined as "the ability of neurons to alter some functional property in response to alterations in input" (50). In this regard, many studies have shown that the brain can change in its structure and function in response to an insult. For example, an increase in the area of the representation zone of the median nerve in the somatosensory cortex has been found after sectioning of the ulnar and radial nerves in monkeys (55). With postnatal median nerve injury alone, however, not only was an increase in the radial representation observed, but also a decrease in the ulnar hand representation (54). Also, cutting the infraorbital nerve prenatally in the rat results in a reduction of the cortical representation of the mystacial vibrissae at the cortex and an increase in the cortical representation of other peripheral receptor surfaces, notably the lower jaw (24). Many other examples of neuroplasticity are cited below.

Normal Brain Development of the Neonatal Rat

At postnatal day $1(\mathrm{Pl})$, differentiation of the neocortical layers is just beginning as seen in the sensorimotor cortex, with only layer I identifiable (17) and cortical lamination is completed by P14 (18). The corpus callosal fibers also continue to develop in the postnatal period. At birth callosal fibers crossed the midline of the brain (56), and after 12 hours postnatally innervate the entire mediolateral extent of the parietal cortex (15). However, reports differ as to when callosal fibers grow into the cerebral cortex, especially the sensorimotor cortex. Some studies show that they do not enter the contralateral sensorimotor cortex until P4 or P5 $(17,55)$. Furthermore, the time of callosal innervation differs for different cerebral cortical areas. It has been found that callosal axons invade the posterior neocortex at P5 (37). This delay in the innervation of the posterior cortex allows the geniculocortical fibres to innervate the posterior cortex at P3/4 prior to the callosal fibres. At birth, thalamocortical afferents are already 
within the upper cortical plate of the somatosensory cortex (48) which may explain the difference in time of callosal innervation between the somatosensory and visual cortices, with thalamocortical afferent growth into the cerebral cortex needing to precede the growth of callosal fibres into the same area. After corpus callosum transection in rats at P2 or P3, morphometric analysis of the primary sensory areas did not show significant differences with respect to cortical depth, total and apical dendritic length and synaptic density (59). Therefore, it appears that early in development the rat brain can compensate for major disruptions in the commissural afferent system.

Effects of Unilateral Frontal Cortical Lesions on the Stucture of the Unablated Hemisphere

Several studies demonstrate that unilateral cortical lesions affect both the ipsilateral hemisphere as well as the contralateral unablated hemisphere, suggesting that the two cerebral hemispheres are dependent on one another.

Effects of Unilateral Frontal Cortical Lesions on the Structure of the Cerebral Cortex

Unilateral frontal cortical lesions introduced at P2 did not affect cortical thickness in the unablated hemisphere (27) although functional recovery did correlate with dendritic morphology in both the unablated and ablated hemisphere (26). When lesions were intoduced at P10, more dendritic spines per unit dendritic length were present at P60 then in animals that had the lesions introduced at $\mathrm{Pl}$, with the $\mathrm{P} 10$ animals performing better in behavioural tests. 
Effects of Unilateral Frontal Cortical Lesions on Cortical Efferents in the Unablated Hemisphere

Motor system plasticity was first demonstrated by Hicks and D'Amato (14) when they showed the formation of a bilateral corticospinal tract (CST) from a single hemisphere following unilateral hemispherectomy in the neonatal rat. Subsequent work in the neonatal rat showed that smaller sensorimotor cortical (SMC) lesions would also induce the formation of an ipsilateral corticospinal projection $(5,8,38,43)$, and these ipsilateral pathways have been suggested to restore some degree of function to the ipsilateral limbs $(2,6)$. Such SMC lesions were performed on postnatal day two (P2), a time when the decussating fibers in the medulla are still less ordered, with a few fascicles or single fibers seen charting abnormal courses away from the principle bundles (45). The adult configuration develops beginning on P3 (46). When SMC lesions were introduced at later ages, after the CST fibers were fully decussated at the medulla, the CST axons innervated the gray matter of the spinal cord bilaterally by recrossing at spinal cord levels rather than by forming bilateral descending tracts (45).

The formation of a bilateral corticofugal pathway is not only restricted to the CST, but aberrant bilateral projections to the red nucleus, superior colliculus, pons and thalamus $(7,8,22,30,58)$ have also been observed after unilateral cortical lesions in the newborn. Further work showed that microstimulation of the unablated hemisphere after unilateral cortical lesions in the neonate elicited ipsilateral limb movements in the adult at stimulus threshold currents lower than those needed to elicit the same movements in adult animals after cortical lesions and unoperated controls (21). Disruption of the low-threshold ipsilateral movements by medullary pyramidotomy suggested that these movements were mediated by the anomalous ipsilateral corticospinal tract fibers which traverse the medullary pyramid (23).

Unilateral SMC lesions have also been shown to cause a change in the topography of the rodent motor cortex. Mapping studies of the cerebral cortex in normal rats (36) demonstrated that the hindlimb area occupies a region immediately caudal to bregma. After neonatal unilateral SMC lesions the unablated hemisphere showed an anterior shift of the motor representation such that both the forelimb and hindlimb areas 
were located rostral to bregma (51). Furthermore, the cortical area caudal to bregma which normally represented hindlimb motor cortex was completely unresponsive to microstimulation.

Subsequent behavioural studies examined the role of the aberrant ipsilateral corticospinal tract in behavior. A head-on placing task of adult rats demonstrated a recovery of deficits induced by unilateral sensorimotor cortical lesions in both adult and neonatal rats (6). However, secondary lesions of the unablated hemisphere revealed deficits in using both forelimbs in those sustaining unilateral cortical lesions as neonates, whereas animals with adult lesions showed a deficit only in using the contralateral limb. Therefore, the formation of a bilateral corticospinal tract may be responsible for recovery of function after neonatal lesions (2). For the adult, however, apparently the mechanism for recovery of function is different as suggested by further experiments Rats that sustained unilateral sensorimotor cortical lesions as neonates showed no preference for either left or right forelimb in a motor task that involved grasping a food pellet, but mature rats that sustained unilateral sensorimotor cortical lesions as an adult showed a preference for the forelimb ipsilateral to the cortical lesion. These findings indicate the presence of a bilateral corticospinal tract in animals that had sustained the cortical lesion as neonates and that it was functional for both forelimbs

Effects of Unilateral Frontal Cortical Lesions on Cortical Afferents in the Unablated Hemisphere

Not much work has been done on changes in cortical afferents in the unablated hemisphere following a unilateral sensorimotor cortical lesion. However, in one study found (49) injection of the unablated hemisphere with a retrograde tracer in adult rats that had sustained the above lesions at $\mathrm{P} 1$ and $\mathrm{P} 30$ resulted in labelling only of the ipsilateral thalamus, and th authors speculated that death of thalamic neurons after neonatal removal of their normal cortical target could be due to their failure to sprout into the opposite cortex because that cortex was already innervated by the remaining thalamus at birth. 
Most studies on the effects of unilateral sensorimotor cortical lesions on cerebral cortical topography have examined the effect of lesions on changes within one modality in different age groups.

\section{Intramodal Effects of Lesions in the Neonate}

Changes in the somatosensory maps deprived of peripheral influences in early development have been observed. Cutting the infraorbital nerve prior to birth in rats results in a reduction of the cortical representation of the mystacial vibrissae and an increase in the cortical representation of other peripheral receptors, most notably from the lower jaw (24).

\section{Intramodal Effects of Lesions in the Adult}

For the motor cortex, it has been found that when areas of the primary motor cortex are disconnected from the periphery following a motor nerve transection in adult rats, these same areas then start to control the movements of different muscle groups (12). This is an orderly reorganization and not random, since only those muscle groups in adjacent areas become represented. This reorganization is evident within hours so new axonal growth and formation of new synaptic connections is unlikely. Rather there could be a change in efficacy of already existing pathways and synapses. The same type of phenomenon has been found for those areas representing the median (54) and radial and ulna hand (55) after injury to the respective nerves in adult monkeys. A model of intracortical connectivity has been proposed in which the organization of the cortical motor map is regulated by inhibitory local circuit neurons (16). Using the GABA antagonist bicuculline methobromide and injecting it into the forelimb area of the primary motor cortex in adult rats, forelimb muscle activity was recorded when a point in the adjacent vibrissae area was stimulated. When injection was terminated, the vibrissae site no longer 
caused forelimb movements. Whether this mechanism applies to neonatal lesions remains to be determined since the latter effects are only tested at least 3-4 months later. It would be necessary to test functional changes very shortly following neonatal lesions and again after a few hours if technically possible.

\section{Cross-modal Effects of Lesions}

A cross-modal effect refers to changes in one modality (e.g., motor system) in response to perturbations (e.g., a lesion) of another modality (e.g., visual system). Examples of this effect are relatively few and primarily involve the visual system. Following monocular enucleation in the neonate, somatosensory responses could be recorded within the visual cortex contralateral to the enucleated eye, thereby demonstrating the expansion of one sensory modality at the expense of another (51). In further work, binocular enucleation resulted in an increase in receptive fields of the barrel cortical cells (53), as well as an expansion in the areas of the cortical barrels (4). Also, auditory cortex neurons show an increased spine density after bilateral enucleation (46). Another example of a cross-modal effect was found following a bilateral lesion of the visual cortex in the adult rabbit (1). The changes in this case were found in the motor cortex. Three different types of cells were recognized in the normal rabbit motor cortex, based on when they were activated during a learning task, including movement of a particular part of the body $(M)$, seizing and/or grinding food (S), and the behavioral phases of the learning process $(L)$. It was found that the number of $S$ units decreased, while the number of $L$ units doubled. A unique example of a cross-modal effect has been demonstrated by ablating the superior colliculus and the lateral geniculate nucleus in the neonatal ferret to induce developing retinal afferents to form connections with the medial geniculate nucleus (44). In the adult, receptive field properties of visual cells in the rewired auditory cortex bore similarities to visual cortical cells recorded in the visual cortex of normal ferrets. Therefore, cortical neurons are by no means hard-wired to their modalities but are influenced by their afferent projections. 
Cross-modal effects are also seen at the subcortical level, particularly at the level of the superior colliculus (34). Ablation of the right eye and right superior colliculus in the neonatal hamster resulted in an increase in the number of cells with receptive fields that extended onto the ipsilateral side of the body in the somatosensory representation in the deep tectal laminae of the left superior colliculus in the adult.

Review of Methodology of Visual Evoked Potentials (VEPS) in Rats

The technique for studying VEPs operates on the principle that the visual signal recorded at the brain surface will always occur at exactly the same time after the visual stimulus. A trigger signal synchronizes stimulus presentation and data collection of EEG (Electroencephalogram). A second flash produces another EEG sample which is added to the previous sample, the two are averaged and so on. Any activity not related to the visual stimulus will not be time locked and will cancel out in the averaging process, leaving a characteristic wave form which represents the massaction response of the recorded area of the visual cortex to the light (Fig. 1). Conventionally, up to 100 responses are averaged together to define the VEP. This procedure is usually carried out in an otherwise dimly lit room, so that the flashing light can exert its maximum effect.

VEPs have been used to map the visual cortex functionally in several studies. The hemispheric distribution of the flash VEPs were found to cover a larger area of the cerebral cortex than that obtained with patterned VEPs (39). Patterned VEP responses were recorded $3.5 \mathrm{~mm}-8.0 \mathrm{~mm}$ posterior to bregma and $1.7 \mathrm{~mm}-5.5 \mathrm{~mm}$ lateral to the sagittal suture. No difference was observed between monocular or binocular stimulation, and this is expected, since in rats the optic system is a predominantly crossed one, and therefore the visual cortex ipsilateral to the stimulated eye receives minimal input. However, some ipsilateral cortical responses have been found using monocular stimulation, and these responses were dependent on the position of the light source relative to the blind axis of the rat and to the position of the reference electrode. Persistence of these responses after cutting the posterior commissural fibers and ipsilateral optic tract suggested that they were in fact volume conducted responses from the 
other hemisphere (39). These small ipsilateral VEP responses have also been found in other studies (52).

The shape of the flash VEP responses obtained was found to vary in different studies. It either consisted of an initial positive (Pl) and then a negative peak (N1), designated as a $P 1 N 1$ waveform (52), or a P1N1P2N2P3N3 waveform (13), or an N1P2N2 waveform (39). The response depends on the laboratory conditions, including light intensity of the stimulus, background illumination, and level of anesthesia. Therefore, any laboratory using evoked potentials needs to establish their own normal values. The actual methodology of recording VEPs in our laboratory is derived from a combination of the protocols used by Onofrj in 1982 and Boyes in 1983 and is explained in more detail in the procedure section.

Review of the Possible Mechanisms of General Cortical Plasticity

Considerable work has been devoted to understanding mechanisms involved in cortical plasticity. That competition for synaptic space is not the only mechanism has been shown with postnatal median nerve injury, where not only was there an increase in the radial hand representation in the sensorimotor cortex of a monkey, but also a decrease in the ulnar hand representation (54). Since the ulnar and radial hand representations are in widely seperated areas of the somatosensory cortex, a mechanism that involves more than simple competition between adjacent representation zones must be involved. When areas of the primary motor cortex were disconnected from the periphery in the adult rat with the transection of the facial nerve in rats, these areas then started to control the movements of different muscle groups (12). That area of the motor cortex that controlled the vibrissae before the transection could control forelimb movements afterwards. However, this reorganization was orderly, representing only those in adjacent areas. This reorganization was evident within hours, and so argues against the possibility of new axonal growth and the formation of new synaptic connections. Rather, there was probably a change in the efficacy of already existing pathways and synapses.

Further evidence for the involvement of preexisting pathways came when using the GABA antagonist bicuculine methobromide (bic) (16). The 
study proposed a model of intracortical connectivity in which the organization of the cortical map is regulated by inhibitory local circuit neurons. Bic was injected into the forelimb area of the primary motor cortex of an adult rat, and recordings were made at a point in the adjacent vibrissae area where vibrissae movements could only be elicited with stimulation before bic application. After bic application, however, this point could elicit both vibrissae and forelimb movements. An attempt to look at the specific patterns of these intrinsic connections between representation zones in the rat motor cortex has been attempted (57). Injections of anterograde tracers were made into the vibrissae region, adjacent forelimb region, and at the border between the two. The labeling of axons within each of the two regions of the axons was restricted within the boundaries of the representation zones. But with injection at the boundary, axons were labeled that distributed radially into both zones. These labeled axons were believed to originate from pyramidal neurons in layers II-VI. So axons from different cell groups appear to overlap, with some groups restricted to zones, and others to borders.

While these intracortical connections are not well characterized, axonal collaterals of pyramidal cells (57) or arbors of thalamocortical axons $(20,31,32)$ may be involved, or involve mechanisms that cause changes beyond the extent of arborization of individual neurons (41). These latter changes may be due to small changes at the brainstem level, which would then be represented over a larger area at the cortical level. The possible neural mechanisms of compensatory plasticity has been reviewed elsewhere (42) and may involve axonal sprouting of intrinsic connections (11). One of the contributing mechanisms thought to occur to result in these plastic changes is an increase in dendritic arbor and dendritic spines in the homotopic area of the unablated hemisphere. These effects were seen better at postnatal day 10 than at postnatal day 1 and in the adult (26). 


\title{
CHAPTER 2
}

\section{MATERIALS AND METHODS}

\begin{abstract}
Animals
A total of 27 Long-Evans, black-hooded rats were divided into 3 groups: 7 adult animals sustaining unilateral SMC lesions as neonates, 6 adult animals sustaining unilateral occipital cortical (OC) lesions as neonates and 14 non-lesion control adult animals .
\end{abstract}

\section{Neonatal Surgery}

Pups at two days of age were anesthetized by hypothermia. Using a dissecting microscope and microsurgical techniques, the scalp was incised, the skull opened and either the sensorimotor or visual cortical area was aspirated unilaterally with gentle suction. The skull flap was then replaced and the skin sutured. The pups were warmed under an incandescent lamp, returned to their mothers until weaning, and then housed individually. Control animals were anesthetized by hypothermia, then warmed under an incandescedent lamp and returned to their mothers.

\section{Flash Visual Evoked Potentials (VEPs)}

At 3-6 months of age, rats were anesthetized with ketamine hydrochloride $(100 \mathrm{mg} / \mathrm{kg})$ and xylazine $(4 \mathrm{mg} / \mathrm{kg})$, administered 
intraperitoneally. Atropine $(160 \mu \mathrm{g} / \mathrm{kg})$ was also administered intraperitoneally as a one time dose to prevent pulmonary edema. During the procedure, supplemental doses of ketamine $\mathrm{HCl}(40 \mathrm{mg} / \mathrm{kg})$ were given as needed. The rat was secured in a stereotaxic frame, the scalp covered with Xylocaine (5\%) ointment, and the skin incised longitudinally along the midline. The cisterna magna was opened to allow the escape of cerebrospinal fluid to reduce the pulsatility of the brain surface. A screw reference electrode was placed $2 \mathrm{~mm}$ rostral and to the right of bregma. The area of skull between the coronal and lambdoid sutures was removed with some of the frontal bone. Throughout the experiment, the surface of the brain was intermittently moistened with physiological saline to prevent drying. A needle ground electrode was inserted into the tail. The active electrode was a stainless steel electrode $0.2 \mathrm{~mm}$ in diameter that was placed on the surface of the dura.

The visual map of the unablated hemisphere was usually recorded using contralateral eye stimulation. However, additional work is also presented using ipsilateral eye stimulation of the unablated hemisphere and recordings from the lesioned hemisphere using both types of stimulation. The light from a Volpi Intalux 6000-1 was directed through a fiber-optic tube and a focusing lens to a uniblitz shutter controlled by a $T 132$ Shutter Driver/Timer (Vincent Assc.). The shutter output was transmitted through a fiber optic tube to the test eye. The other eye was exposed to constant low background illumination. Stimulus flashes were delivered at a frequency of $1 \mathrm{~Hz}$, with an exposure time of $151 \mathrm{~ms}$ and a delay of $900 \mathrm{~ms}$. Responses were differentially amplified $(0.1-200 \mathrm{hz})$, averaged $(\mathrm{N}=100)$, and stored using a Nicolet Pathfinder signal averaging system. The recording electrode was moved systematically in $1-2 \mathrm{~mm}$ steps to define the boundaries of the visual cortex. At each point, recordings were made two to six times and results superimposed to determine the reproducibility of the response. The boundary was defined as the point where no reproducible signal was obtained. Where maximal responses were recorded, the recording electrode was moved at $0.5 \mathrm{~mm}$ steps around this area. 


\section{Intracortical Microstimulation}

After VEP recording the dura mater was carefully incised and displaced both laterally and medially to expose the underlying brain. A glass coated sharpened tungsten wire with a $100 \mu \mathrm{m}$ non-coated tip was used as the stimulating electrode. The electrode was placed into the cerebral cortex at a depth of $1.7 \mathrm{~mm}$ and $100 \mu \mathrm{A}$ of direct negative current was applied through the electrode as a $300 \mathrm{msec}$ train of $250 \mu \mathrm{sec}$ pulses at $300 \mathrm{~Hz}(36)$, using the Grass $\$ 48$ stimulator by way of a photoelectric stimulus isolation unit (Grass Instrument Co.). Limb movements were observed and recorded for each stimulation point. Microstimulation was carried out at $1 \mathrm{~mm}$ divisions from the midline and began lateral to bregma. Stimulations were delivered at $1 \mathrm{~mm}$ intervals in a rostral to caudal direction and terminated when two adjacent stimulation points gave no response. Only the caudal area of the motor cortex was mapped.

\section{Tissue Collection and Preparation}

After the electrophysiological studies were completed, the rats were injected with an overdose of sodium pentobarbital $(84 \mathrm{mg} / \mathrm{kg})$ and perfused intracardially with a formaldehyde fix solution (1.3\%). The brains were removed, postfixed, cryoprotected and stored at $-70^{\circ} \mathrm{C}$, then sectioned coronally or sagittally at $40 \mu \mathrm{m}$ on a cryostat. Transverse sections of the brainstem and coronal sections of the brain were stained with the Klüver method (25). Sagittal sections of the brain were stained either with the Klüver method or with toluidine blue (9).

\section{Data Analysis}

The VEP maps in the following figures were made by first normalizing the VEP amplitudes obtained, expressing each as a percentage of the response maxima for each rat. The values were thenaveraged together at each point on the cortical surface relative to bregma. The averaged results for each point were used to plot a contour map joining points of 
equal amplitude (Trimetrix Axum Program). The experimental results were statistically compared to ice controls using two parameters: (1) The rostralcaudal position relative to bregma at which reproducible VEP responses could no longer be obtained as recordings were made progressively further from the response maxima, and (2) the rostral-caudal position relative to bregma of the response maxima. The values obtained were compared using an unpaired two-tail t-test (Microsoft Excel software version 4.0). For the motor maps, each point of the motor cortex represented in the following diagrams shows the movement on microstimulation that was elicited in the majority of rats tested. 


\section{CHAPTER 3}

\section{RESULTS}

\section{Electrophysiological Analysis of Control Rats}

Three major peaks, an initial positive deflection (P1), followed by a negative deflection $(N)$, and then a second, broader positive deflection (P2), were observed in the VEP response in normal rats (Fig. 1A). As the electrode moved away from the response maxima additional peaks usually appeared. To measure VEP amplitude we noted the latency at which the maximum amplitude response occurred, and then in subsequent VEPs elsewhere in the cortex of the same hemisphere the amplitudes of the peaks closest to this latency were measured. The amplitude noted in our studies was B-Pl since it was easier to measure as one progressed away from the maximum. This can be seen from Fig. 1d, where $N$ and P2 cannot be discerned from the surrounding noise. We chose a window in which to accept peaks, and any peaks beyond $+/-20 \mathrm{~ms}$ of that found at the maximum were disregarded. Table 1 shows the range of normal values of the $\mathrm{Pl}$ absolute latency and B-P1 amplitude. There was no significant difference between contralateral and ipsilateral eye stimulation.

When recording from the hemisphere contralateral to the stimulated eye, the boundary of the area from which reproducible responses could be obtained ran rostro-laterally, with its maximum rostral extent at $3 \mathrm{~mm}$ caudal to bregma (Fig. 2A, 5\% line). The response maxima were located $\sim 6 \mathrm{~mm}$ caudal to bregma and $\sim 3.5 \mathrm{~mm}$ lateral to bregma. VEPs recorded from the hemisphere ipsilateral to the eye being stimulated were more variable in amplitude and on average gave responses of lower amplitude at a particular point. 
Table 1.- Comparison of VEP Results From Normal Rats Using Contralateral and Ipsilateral Eye Stimulation With Respect to the Hemisphere Recorded

\begin{tabular}{lrc}
\hline & Latency (ms) & Amplitude (UV) \\
\hline \hline Contralateral Eye Stimulation $(n=8)$ & $108.0+/-24.20$ & $136.1-406.2$ \\
Ipsilateral Eye Stimulation $(n=5)$ & $86.4+/-11.84$ & $35.3-351.1$ \\
& & \\
\hline
\end{tabular}

Notes: Latencies are expressed as the mean +/- standard deviation, and the amplitude as a range, and are the values obtained at the response maxima for each hemisphere. There was no significant difference in latency and amplitude between the two types of stimulation, using a $5 \%$ significance level. For latency an unpaired two-tail t-test was used, and for amplitude a Mann-Whitney two-tail U-test.

The caudal area of the motor map represented by hindlimb and forelimb movements evoked by intracortical microstimulation extended back to $3.5 \mathrm{~mm}$ caudal to bregma and $0.5-3.5 \mathrm{~mm}$ lateral to the midline, with a small area within the region mapped showing forelimb responses. No effort was made to define the rostral border of the motor cortex. This agrees with previously published results $(36,50)$.

\section{Analysis of the SMC Lesions}

The typical location of a SMC lesion is presented in Fig 3A. Most of the sensorimotor cortex is absent, with the underlying structures still intact. In all cases the caudate-putamen and the hippocampus and fimbrae were present (Fig. 3B), In some specimens, the rostral part of the occipital cortex and part of the alveus of the hippocampus were absent. Atrophy of the corresponding pyramidal tract (Fig. 3C) indicated completeness of the sensorimotor cortical lesion. Fig. 4A superimposes the outlines of the SMC lesions. 


\section{Electrophysiological Analysis of Rats with SMC Lesions}

Fig. 2B shows the average results of VEPs and microstimulation responses in the SMC lesioned rats, with the unablated hemisphere recorded during contralateral eye stimulation and the lesioned hemisphere during ipsilateral eye stimulation. In the unablated hemisphere, the motor map has shifted $2 \mathrm{~mm}$ rostrally and has increased in width, extending from $0.5 \mathrm{~mm}$ to $4.5 \mathrm{~mm}$ from the midline. Using contralateral eye stimulation, the $5 \%$ line of the visual map in the unablated hemisphere spread rostrally by up to $4 \mathrm{~mm}$. The location of the maximum amplitude moved only minimally rostrally to $5.5 \mathrm{~mm}$ caudal to bregma; nonetheless this was significant ( $t=2.7$, $\mathrm{p}<0.01$ ).

To compare the visual maps obtained in normal and lesioned animals, we measured the boundary position at which a reproducible VEP was no longer obtained as the electrode position was moved rostrally from the response maxima and we also measured the rostral-caudal position where the maximal response was obtained (Table 2 and Fig. 2). When recording from the unablated hemisphere using contralateral eye stimulation, significant differences were obtained at $3 \mathrm{~mm}$ lateral to bregma. The maximum point shifts significantly rostrally but less than the border.

A comparison can be made between the unablated hemisphere of the SMC lesioned and normal animals by comparing the $5 \%$ boundaries of the visual maps for each rat and superimposing them on the same map (Fig. 2C). The results from the lesioned animal are seen to be scattered more rostrally then those from the normal animals, with some overlap. When the ablated hemisphere was looked at using ipsilateral eye stimulation, we observed that VEP responses also showed a significant rostral shift at $3 \mathrm{~mm}$ and $5 \mathrm{~mm}$ lateral to the midline (Fig. 2B and Table 2), with the response maxima also showing some shift rostrally. The results from the normal rats (Fig. 5) show no correlation between contralateral and ipsilateral eye stimulation $\left(r^{2}=0.14\right)$ as far as the position of where VEP responses were no longer recordable are concerned. When similar results obtained as in Fig. 2B from three SMC lesioned rats were plotted, they all lay on a perfect straight line $\left(r^{2}=1.0\right)$, showing perfect correlation. 
When the unablated hemisphere was recorded using ipsilateral eye stimulation (Fig. 2D) significant rostral shifts of the visual map were seen at $3 \mathrm{~mm}$ lateral to bregma, with no change in the position of the maximum response. Recording from the ablated hemisphere using contralateral eye stimulation revealed a significant difference with a rostral shift of the visual map and a shift in the maximum point.

\section{Analysis of the Electrophysiological Studies from OC Lesioned Rats}

The visual cortex in the unablated hemisphere was also mapped in adults that sustained unilateral $O C$ lesions as neonates. The outlines of all the $O C$ lesions superimposed on one outline of the mature rat brain are shown diagramatically in Fig. $4 \mathrm{~B}$. The VEPs from the $\mathrm{OC}$ lesioned animals were found not to differ significantly from normal, both in the boundary of the visual cortex and the position of the response maxima (Fig. 6). Fig. 6A shows the results from all six $O C$ lesioned rats. When the $5 \%$ boundaries from each of the rats were drawn, we noticed one animal where the boundary extended as far as $2 \mathrm{~mm}$ rostral to bregma (Fig. 6B). When this outlier was removed from the overall result (Fig. 6C), the results still showed no significant difference from normal. This lesion is indicated by a dotted line in Fig. 4B. When coronal sections of this brain were taken, the lesion was seen to interfere with fibers running through the splenium of the corpus callosum (Fig. 7).

\section{Histology of the Visual and Motor Maps in Normal and SMC Lesioned Rats}

When sagittal sections of the brains from one normal and the unlesioned hemisphere of one SMC lesioned rats were compared (Fig. 8), no difference was seen in the histology of the sections, despite the functional shift of the border between visual and motor cortex. As can be seen from Fig. $8 \mathrm{~A}$, the visual responses end $\sim 2 \mathrm{~mm}$ rostral to the cytoarchitectural border between the visual and sensory cortices in a hemisphere from a control rat. When the unablated hemisphere from an 
Table 2.-Summary of the statistical analyses comparing the rostral borders of the visual maps of lesioned rats and their VEP maxima to controls using contralateral eye stimulation.

(a) SMC lesioned brain, unablated hemisphere

\begin{tabular}{cclcl}
$\begin{array}{l}\text { Lateral to } \\
\text { midline }(\mathrm{mm})\end{array}$ & $\begin{array}{l}\text { Mean rostral border } \\
+/ \text { - standard deviation }\end{array}$ & $\begin{array}{l}\text { Control } \\
\text { values }\end{array}$ & $\begin{array}{l}\dagger \\
\text { value }\end{array}$ & $\begin{array}{l}\mathrm{P} \\
\text { value }\end{array}$ \\
\hline \hline 1 & $-2.50+/-1.73$ & $-4.00+/-1.26$ & 1.34 & $>0.05$ \\
3 & $-0.57+/-1.51$ & $-2.57+/-0.65$ & 3.35 & $<0.01$ \\
5 & $-1.80+/-1.3$ & $-2.25+/-1.86$ & 0.57 & $>0.05$ \\
Maxima & $-4.86+/-0.9$ & $-5.93+/-0.76$ & 2.7 & $<0.05$ \\
\hline
\end{tabular}

(b) SMC lesioned brain, ablated hemisphere

\begin{tabular}{ccccc}
$\begin{array}{c}\text { Lateral to } \\
\text { midline }(\mathrm{mm})\end{array}$ & $\begin{array}{c}\text { Mean rostral border } \\
+/ \text {-standard deviation }\end{array}$ & $\begin{array}{l}\text { Control } \\
\text { values }\end{array}$ & $\begin{array}{l}t \\
\text { value }\end{array}$ & $\begin{array}{l}\mathrm{P} \\
\text { value }\end{array}$ \\
\hline \hline 3 & $-2.25+/-0.5$ & $-2.57+/-0.65$ & 7.62 & $<0.001$ \\
4 & $-0.75+/-0.5$ & $-2.50+/-0.76$ & 4.6 & $<0.001$ \\
Maxima & $-4.50+/-1.0$ & $-5.93+/-0.76$ & 2.65 & $<0.05$ \\
\hline
\end{tabular}

\section{(c) OC lesioned brain}

\begin{tabular}{ccccc}
$\begin{array}{l}\text { Lateral to } \\
\text { midline }(\mathrm{mm})\end{array}$ & $\begin{array}{c}\text { Mean rostral border } \\
+/ \text {-standard deviation }\end{array}$ & $\begin{array}{c}\text { Control } \\
\text { values }\end{array}$ & $\begin{array}{l}\dagger \\
\text { value }\end{array}$ & $\begin{array}{l}\mathrm{p} \\
\text { value }\end{array}$ \\
\hline 1 & $-3.25+/-1.26$ & $-4.00+/-1.26$ & 0.92 & $>0.05$ \\
3 & $-2.20+/-0.84$ & $-2.57+/-0.65$ & 0.89 & $>0.05$ \\
5 & $-2.25+/-0.96$ & $-2.25+/-1.86$ & 0.0 & $>0.05$ \\
Maxima & $-5.50+/-1.41$ & $-5.93+/-0.76$ & 0.65 & $>0.05$ \\
\hline
\end{tabular}

Note: Studies from lesioned animals were compared to control results using an unpaired two-tail t-test. 
Table 3.-Summary of the statistical analyses comparing the rostral borders of the visual maps of lesioned rats and their VEP maxima to controls using ipsilateral eye stimulation.

(a) SMC lesioned brain, unablated hemisphere

Lateral to Mean rostral border Control $t \quad p$

midline $(\mathrm{mm})+/$-standard deviation values value value

\begin{tabular}{cllll}
\hline \hline 1 & $-6.33+/-1.53$ & $-6.40+/-1.95$ & 0.06 & $>0.05$ \\
3 & $-3.50+/-1.73$ & $-6.17+/-1.83$ & 2.34 & $<0.05$ \\
5 & $-6.33+/-0.58$ & $-6.40+/-1.52$ & 0.09 & $>0.05$ \\
Maxima & $-6.25+/-0.5$ & $-6.60+/-1.52$ & 0.48 & $>0.05$ \\
\hline
\end{tabular}

(b) SMC lesioned brain, ablated hemisphere

\begin{tabular}{ccccc}
$\begin{array}{l}\text { Lateral to } \\
\text { midline }(\mathrm{mm})\end{array}$ & $\begin{array}{c}\text { Mean rostral border } \\
+/ \text {-standard deviation }\end{array}$ & $\begin{array}{c}\text { Control } \\
\text { values }\end{array}$ & $\begin{array}{l}\dagger \\
\text { value }\end{array}$ & $\begin{array}{l}\mathrm{p} \\
\text { value }\end{array}$ \\
\hline \hline 1 & $-2.67+/-2.08$ & $-6.40+/-1.95$ & 2.51 & $<0.05$ \\
3 & $-1.00+/-1.22$ & $-6.17+/-1.83$ & 5.59 & $<0.001$ \\
5 & $-3.67+/-2.08$ & $-6.40+/-1.52$ & 1.98 & $>0.05$ \\
Maxima & $-4.50+/-1.12$ & $-6.60+/-1.52$ & 2.49 & $<0.05$ \\
\hline
\end{tabular}

(c) OC lesioned brain

\begin{tabular}{ccccc}
$\begin{array}{l}\text { Lateral to } \\
\text { midline }(\mathrm{mm})\end{array}$ & $\begin{array}{c}\text { Mean rostral border } \\
+/ \text {-standard deviation }\end{array}$ & $\begin{array}{c}\text { Control } \\
\text { values }\end{array}$ & $\begin{array}{l}\dagger \\
\text { value }\end{array}$ & $\begin{array}{l}\mathrm{P} \\
\text { value }\end{array}$ \\
\hline \hline 1 & $-6.33+/-1.15$ & $-6.40+/-1.95$ & 0.06 & $>0.05$ \\
3 & $-5.60+/-1.34$ & $-6.17+/-1.83$ & 0.60 & $>0.05$ \\
5 & $-5.80+/-1.10$ & $-6.40+/-1.52$ & 0.72 & $>0.05$ \\
Maxima & $-6.25+/-0.96$ & $-6.60+/-1.52$ & 0.42 & $>0.05$ \\
\hline
\end{tabular}

Note: Studies from lesioned animals were compared to control results using an unpaired two-tail t-test.

SMC lesioned animal is looked at (Fig. $8 \mathrm{C}$ ), the visual responses end $\sim 6 \mathrm{~mm}$ rostral to this border. 


\section{CHAPTER 4}

\section{DISCUSSION AND CONCLUSION}

\section{Summary of the Results}

Our results indicate that functional visual cortical topography shows a reorganization in both the ablated and unablated hemispheres of mature rats following unilateral neonatal SMC lesions. This change was not accompanied by a histological alteration in the cytoarchitecture of the cerebral cortex based on Nissl stain analysis. Furthermore, such a reorganization was not apparent with unilateral neonatal OC lesions.

\section{A Comparison of our Results to Past Findings}

\section{Possible Mechanisms of Neuroplasticity}

Our observation that the functional visual topography showed a reorganization without an apparent change in cytoarchitecture of the cerebral cortex supports the theory that changes in cortical topography in response to cortical lesions are due to latent intracortical connections becoming functional $(16,42)$. One can consider the cerebral cortex as being in equilibrium in that, while some neurons remain active in response to excitatory neurotransmitters, others remain latent. A lesion in one part of the cerebral cortex, or of afferents and efferents of the cerebral cortex, may upset this equilibrium. One may therefore envision a scenario whereby the cerebral cortex, while trying to compensate for the lesion, somehow 
shifts the balance between inhibition and excitation in the cerebral cortex, allowing latent connections to now become functional.

We need, however, to explain the observed phenomenon of why unilateral SMC lesions, and not OC lesions, cause the above changes. Our observation that a unilateral $\mathrm{OC}$ lesion with a disruption of the corpus callosum causes a reorganization of the visual cortex in the unablated hemisphere would indicate that the corpus callosum plays some role. In our review of the literature on normal development of the rat brain (see section 2.2.) we discovered that at the time of our neonatal lesions at P2, the SMC is innervated by callosal fibres, whereas the OC has yet to recieve these fibres. We therefore propose the following theory as to the difference, as depicted in figure 9. The destruction of callosal fibres running from the ablated to the unablated hemisphere would not be responsible for the changes seen, since these would be involved in both the sensorimotor and all the occipital cortical lesions (Fig. 9). However, the callosal fibres running from the unablated to the ablated hemisphere would not be affected by occipital lesions since the fibres have not yet innervated the visual cortex, although they would be affected by that occipital lesion located in the posterior midline location. This hypothesis would need further work to verify since our work has not attempted to find the mechanisms of these changes. The loss of callosal cell bodies may therefore lead to the above changes in the neurotransmitter pool in the cerebral cortex. Furthermore, direct projections from the visual cortex to the medial area of the primary motor cortex (33) may also influence the movement of the visual with the motor cortex. Therefore a change in the topography of either the visual or motor cortex could influence the other through these interconnections.

Earlier work showed that cutting the corpus callosum does not change the cytoarchitecture of the cerebral hemispheres (59) and so a loss of callosal activity may contribute to the above changes. If we had seen a change in cytoarchitecture in our brain sections, then a mechanism other then that involving the corpus callosum would have had to be looked for. 


\section{Cross-modal Neuroplasticity}

Different forms of neuroplasticity have been reported in the literature ranging from within one modality to between modalities $(5,7,8,22,24,38,43,53,54)$. From our work it appears that with unilateral lesions, the unablated hemisphere will only respond to the lesion if the corpus callosum is involved. We believe that these different forms of changes perhaps operate with the same mechanism but on different levels. For example, the lowest level is demonstrated when areas of the primary motor cortex are disconnected from the periphery following a motor transection in adult rats, and these same areas then start to control the movements of different muscle groups (12). Observing these changes just in the motor cortex indicates that the neurotransmitter pool may be controlled at a local level. With larger lesions of the cortex, as in our study, higher levels come into play since now the whole motor cortex is involved.

We therefore have what appears to be tiers of influence of CNS lesions on cortical topography. The first tier is shown by changes within a single modality. The second tier is shown by cross-modal changes within the same hemisphere. The third tier is shown by intramodal changes that manifest in the unablated hemisphere. Finally, we have the tier where cross-modal changes are shown in the unablated hemisphere. As one goes up this tier system, more of the cerebral cortex is involved in the insult to the CNS.

Plasticity of the Optic Pathway

In normal rats the anterior borders of the visual maps of ipsilateral and contralateral eye stimulation did not correlate when stimulating the same eye (Fig. 9). This seems to argue against the ipsilateral responses being volume-conducted responses from the contralateral hemisphere, as has been claimed elsewhere (40); we would expect a larger ipsilateral response with a larger contralateral response if this was the case. The results from the SMC lesioned animals, however, correlated perfectly. We can hypothesize from this that in normal rats the ipsilateral pathway can generate VEPs, but not consistently. These responses are variable since it 
depends on how much the ipsilateral pathway is left after retraction during development $(28,29)$. In the SMC lesioned rats, the ipsilateral pathway may be more persistent either due to failure to retract, or by the formation of atypical uncrossed branches at the optic chiasm (10). This aberrant pathway development would depend on how much influence the unilateral SMC lesion has on the visual cortex, which then manifests in both hemispheres. Further work is needed to verify the above hypothesis. The persistence or formation of the ipsilateral optic pathway has been shown with many brain lesions, such as neonatal enucleation $(10,19,28,29)$ and with neonatal optic tract lesions (40).

\section{Future Work}

We have examined the effects of unilateral SMC lesions on the visual system, but other modalities may also be examined in the same way. The somatosensory cortex may be mapped using short latency somatosensory evoked potentials by stimulating the median or ulna nerve at the wrist, and the tibial nerve at the ankle. Also, we have speculated that the difference in the results seen between SMC and OC lesioned animals was that the occipital cortex at the time of the lesion (P2), was not yet innervated by callosal fibres. To test this, we can introduce the OC lesions at P7, a time when the occipital cortex will have its innervation of callosal fibers. The results should then be the same as for the SMC lesions if we are correct. We may also do the above by introducing lesions in the adult and see if the effects are as pronounced. We looked at the effects of our unilateral SMC lesions after three months. But it may be possible that the changes may commence even hours after the lesion, and a time course study would also be informative as to the mechanism of the changes. The involvement of the corpus callosum may be tested by cutting the corpus callosum at the midine at P2 instead of introducing unilateral lesions. We may also look to see if the afferent connections of the cortex to the thalamus have changed in any way by injecting retrograde tracers into the newly postitioned visual cortex. Finally, if we believe the assumption that neuroplasticty occurs to allow the organism to survive amongst the fittest with a CNS insult, has the visual system in the rat been enhanced in some 
way with the reorganization of the visual cortex we observed. Further experiments can test the visual acuities of our lesioned animals or their reflexes to visual cues.

\section{Conclusion}

We have shown a change in cortical visual topography in the unlesioned hemisphere in the adult rat following a unilateral sensorimotor cortical lesion in the neonate. The rostral movement of the visual cortex appeared to parallel that of the motor cortex. This functional plasticity was not accompanied by any change in the cytoarchitecture of the cerebral cortex. These results are a further demonstration of the capacity for the developing CNS to functionally reorganize following neonatal brain damage. 
APPENDIX

Figures 1-9 
Figure 1. A comparison of VEPs obtained at different points on one hemisphere in a normal rat using contralateral eye stimulation. a VEP from near the response maxima. Two trials from the same point on the cerebral cortex are shown superimposed, showing the reproducability of the response. The arrow indicates the time at which the flashing light is presented to the rat's visual field. The vertical bar represents $50 \mu \mathrm{V}$ and the horizontal bar $30 \mathrm{~ms}$. $\mathrm{B}=$ baseline point, $\mathrm{Pl}=$ first positive peak, $\mathrm{N}=$ negative peak and $\mathrm{P} 2=$ second positive peak, sweep $=300 \mathrm{~ms}$. b-e Responses obtained from positions progressively more rostral then a ( $1 \mathrm{~mm}$ intervals), until no reproducible response was recorded at $\mathbf{e}$. $\mathrm{Pl}$ in $\mathrm{a}$ is the point of maximum amplitude (at $51.2 \mathrm{~ms}$ ), and the amplitudes of the remaining responses were taken from the baseline to the first positive peak that had a latency closest to $51.2 \mathrm{~ms}$ 

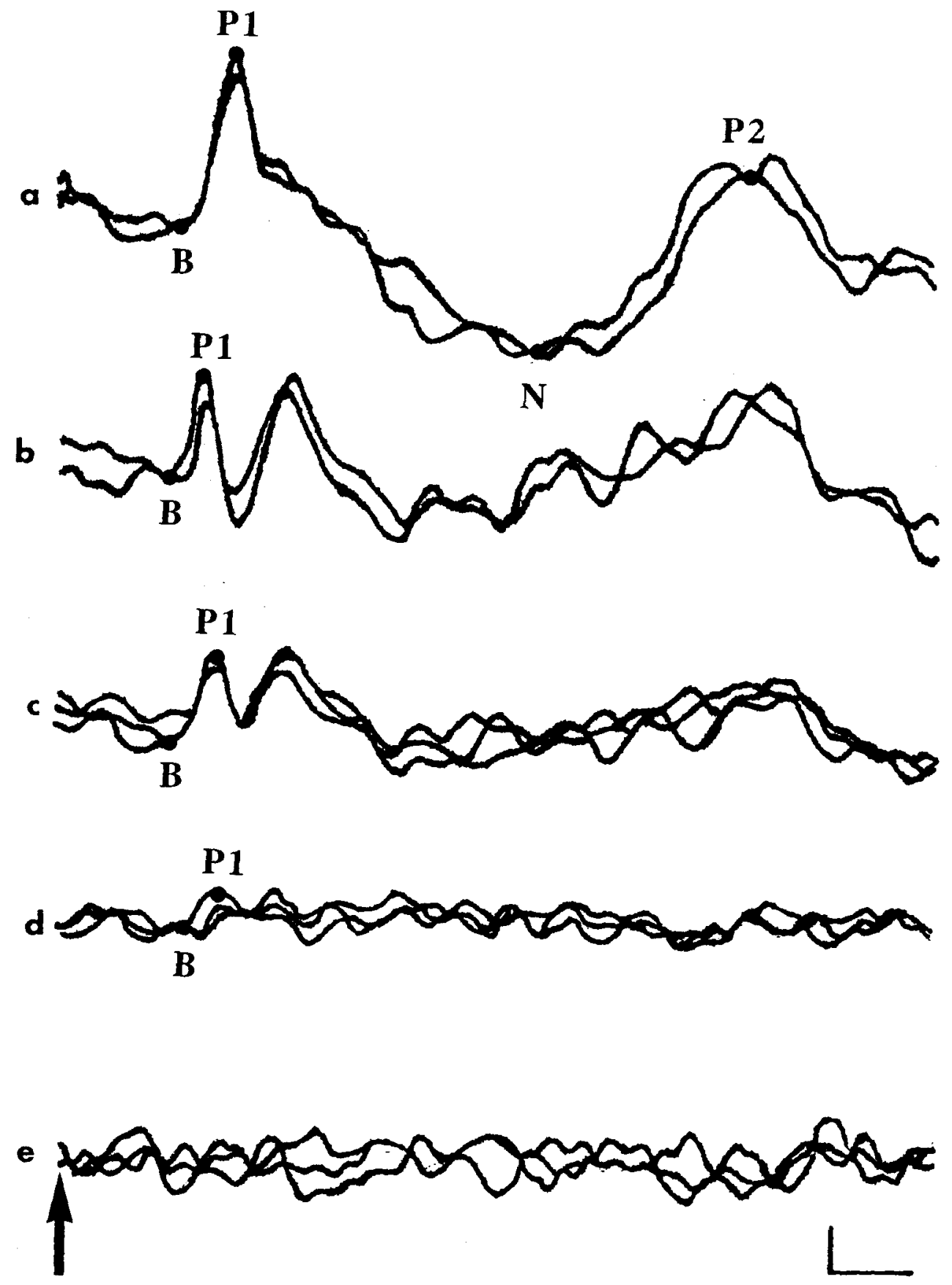
Figure 2.Visual and motor maps of control and SMC lesioned rats (A) Representation of the visual and motor map of control rats. The terms contralateral and ipsilateral refer to the orientation of the hemisphere with respect to the eye being stimulated during VEP recording. The visual map is represented by the continuous lines, and the percentages are that of the maximum response obtained in each rat in the contralateral hemisphere. The lines pass through points of equal percentage values, with the $5 \%$ line shown in bold. The contralateral visual map is the average result of fourteen rats, and the ipsilateral visual map the average result of six rats. The motor map, represented by the discontinuous line, was determined using microstimulation and shows the maximum extent of responses obtained from the results of four rats. (B) Representation of the visual and motor maps of mature rats that had sustained unilateral sensorimotor cortical lesions as neonates. The contralateral visual map is the average result of seven rats and the ipsilateral visual map the average result of five rats. The motor map shows the maximum extent of recordable responses from the results of six rats. (C) The $5 \%$ boundaries of the visual maps of normal (solid line) and SMC lesioned rats (dashed line) using contralateral eye stimulation of each of the rats tested are shown. The results from the lesioned animal are from the unablated hemisphere. (D) The eye contralateral to the lesioned hemisphere is stimulated. $H=h i n d l i m b$ area, $\mathrm{H} / \mathrm{F}=$ hindlimb and forelimb area. The statistical results for $(B)$ and (D) that are summarized in Table 2 are shown here with the statistical comparisons of the border of the visual map to the normal map in (A) shown along the top near the $5 \%$ border, and the statistics of the response maxima indicated with the letter $\mathrm{M}$ at the bottom of each map. The points lateral to the midline at which the statistics are shown in this figure are indicated by arrows along the horizontal scale bar at the top of each figures (B) and (D). ${ }^{*}=\mathrm{P}<0.05,{ }^{* *}=\mathrm{P}<0.01,{ }^{* * *}=\mathrm{P}<0.001, \mathrm{~ns}=$ not significant. 

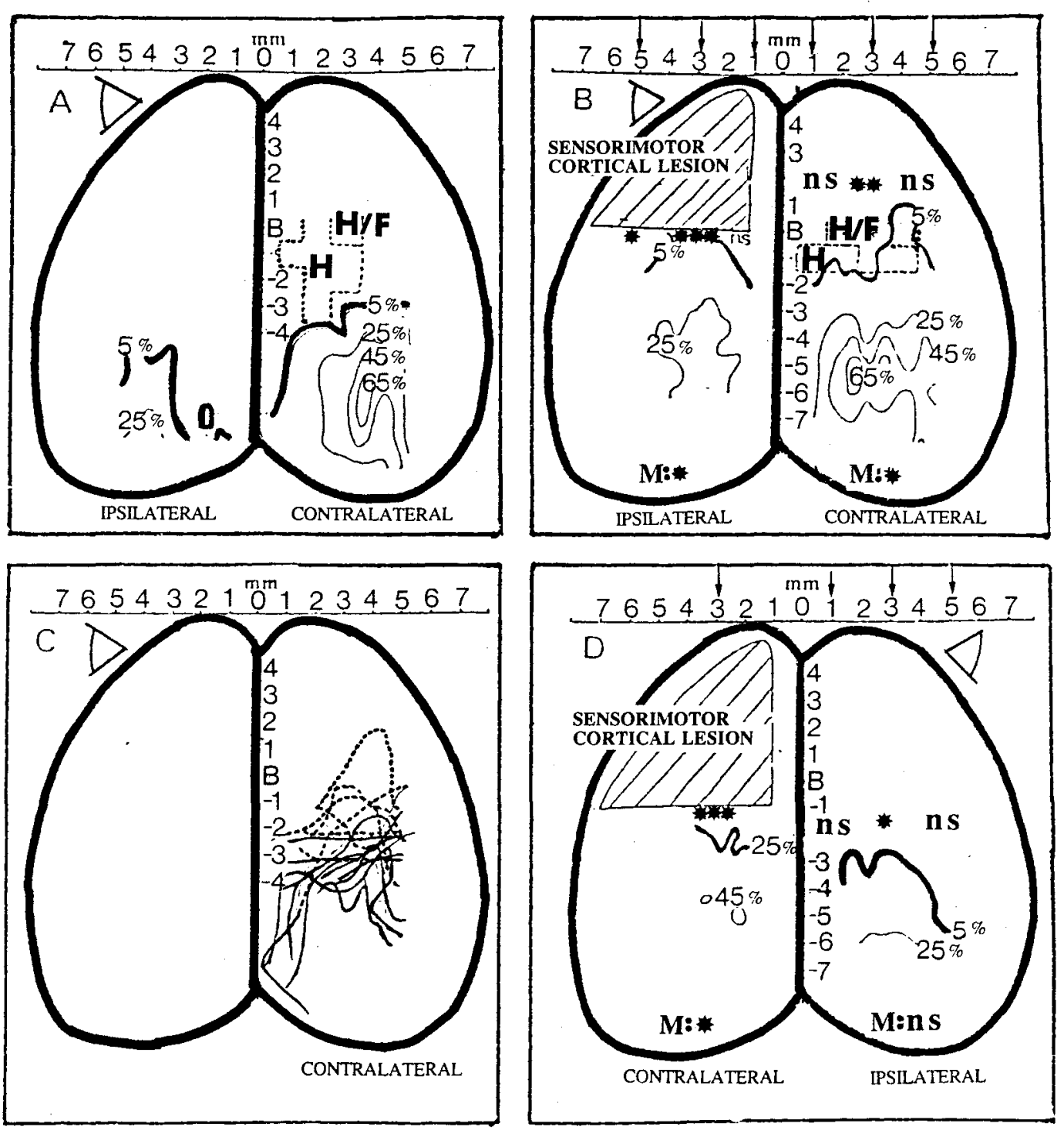
Figure 3. Gross and microscopical analyses of SMC lesioned rat brains. (A) Photograph of a brain from a mature rat that had sustained a unilateral SMC lesion (indicated by an asterisk) as a neonate. Scale bar $=400 \mu \mathrm{m}$.

(B) Photomicrograph of a sagittal section of the lesioned hemisphere of a brain from a mature rat that had sustained a unilateral SMC lesion as a neonate, showing the extent of the lesion (Klüver stain). The top of the figure is the dorsal end of the section and the rostral end is on the left. $\mathrm{RF}=$ rhinal fissure, $\mathrm{LO}=$ lateral orbital cortex fmi=forceps minor corpus callosum, $\mathrm{CPu}=$ caudate putamen, $\mathrm{Oc} 2 \mathrm{MM}=0 \mathrm{cc}$ ipital cortex, area 2, CA1/ CA3=hippocampal fields, $D G=$ dentate gyrus, fi=fimbria hippocampus. Scale bar $=800 \mu \mathrm{m}$.

(C) Photomicrograph of a transverse section of the brainstem at the level of the facial nucleus, from a mature rat that had sustained a unilateral SMC lesion as a neonate. The atrophic pyramidal tract is on the left (Klüver stain). PT=intact pyramidal tract, scale bar $=400 \mu \mathrm{m}$. 

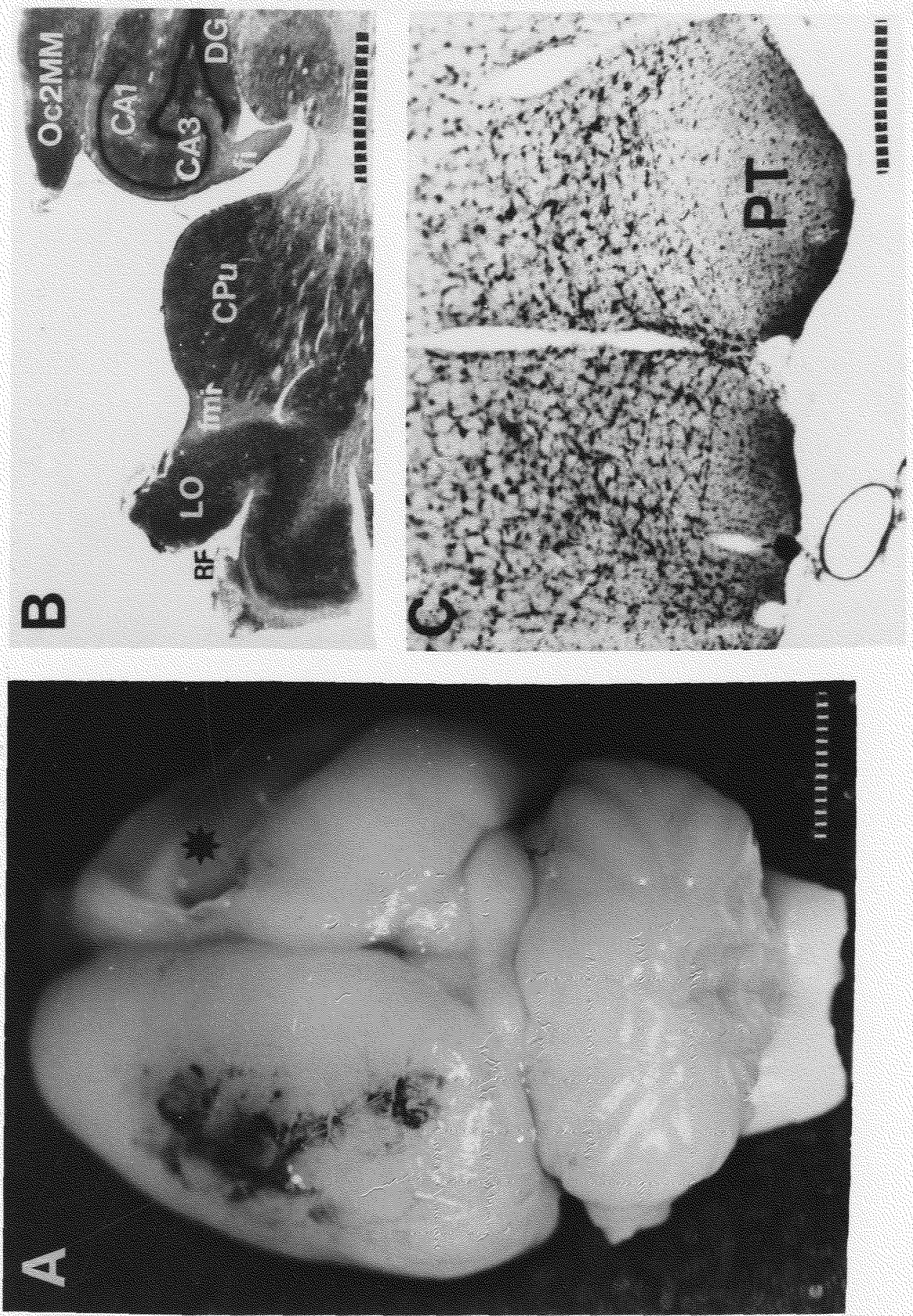
Figure 4. The outlines of (A) unilateral SMC lesions of seven rats and (B) unilateral $O C$ lesions of six rats are shown superimposed. The dashed line in (B) represents an $O C$ lesion that gave a unique result as explained in the text. 


$$
1000
$$


Figure 5. Regression plots for normal (filled circles) and SMC lesioned (open circles) rats where the most rostral point of the visual map using ipsilateral eye stimulation is plotted against the results obtained using contralateral eye stimulation, both methods stimulating the same eye for each rat. For the SMC lesioned animals, the results from the unablated hemisphere are shown. 


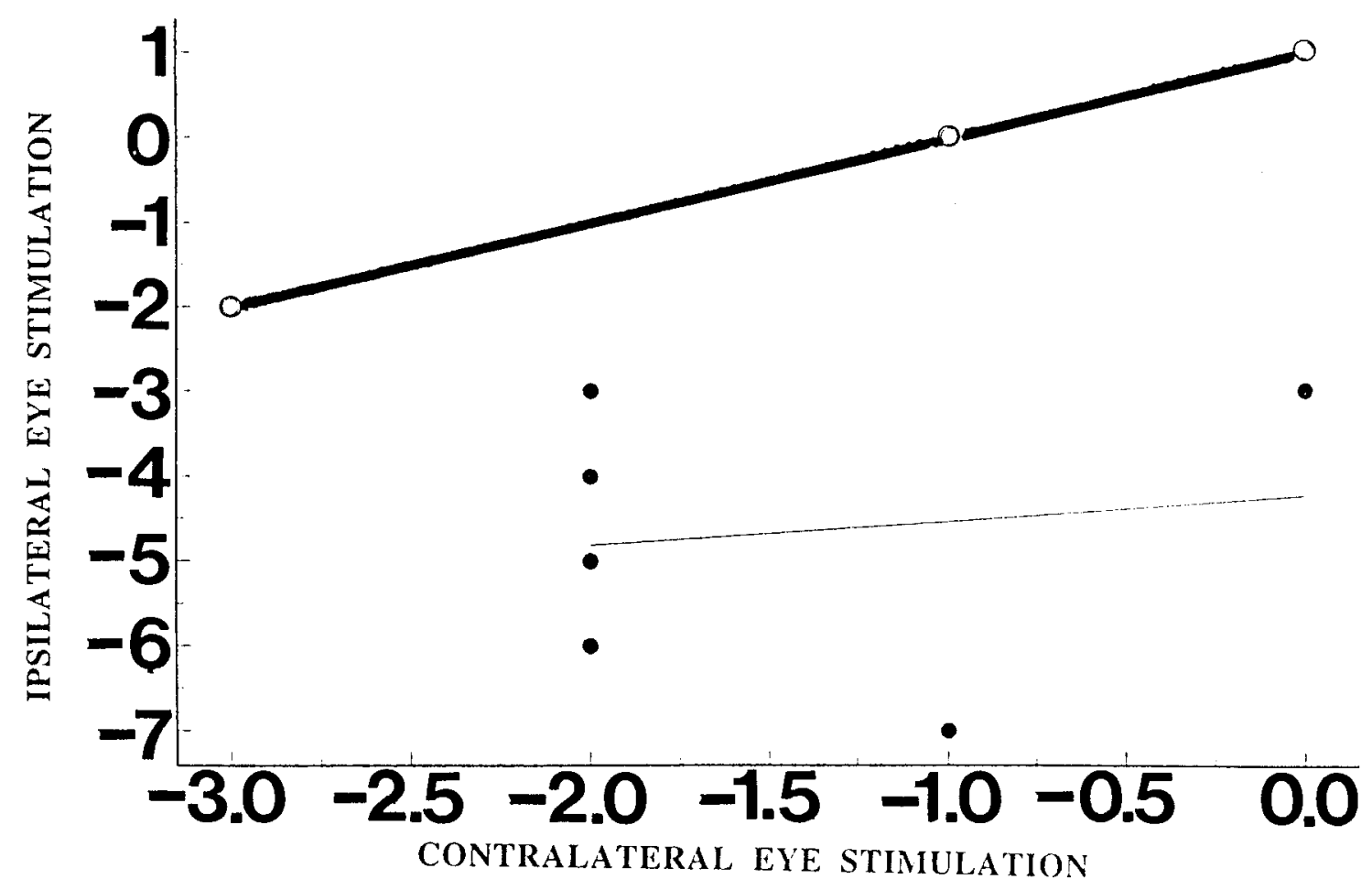


Figure 6. Representation of the visual maps of mature rats that had sustained unilateral $O C$ lesions as neonates. All results are from the unablated hemisphere. (a) Averaged visual map from six rats using contralateral eye stimulation, (b) $5 \%$ boundaries of the visual maps from each of the six $O C$ rats using contralateral eye stimulation,

(c) averaged visual map of the OC lesioned rats with the outlier result shown in (b) as $\S$ removed, (d) averaged visual map using ipsilateral eye stimulation. 

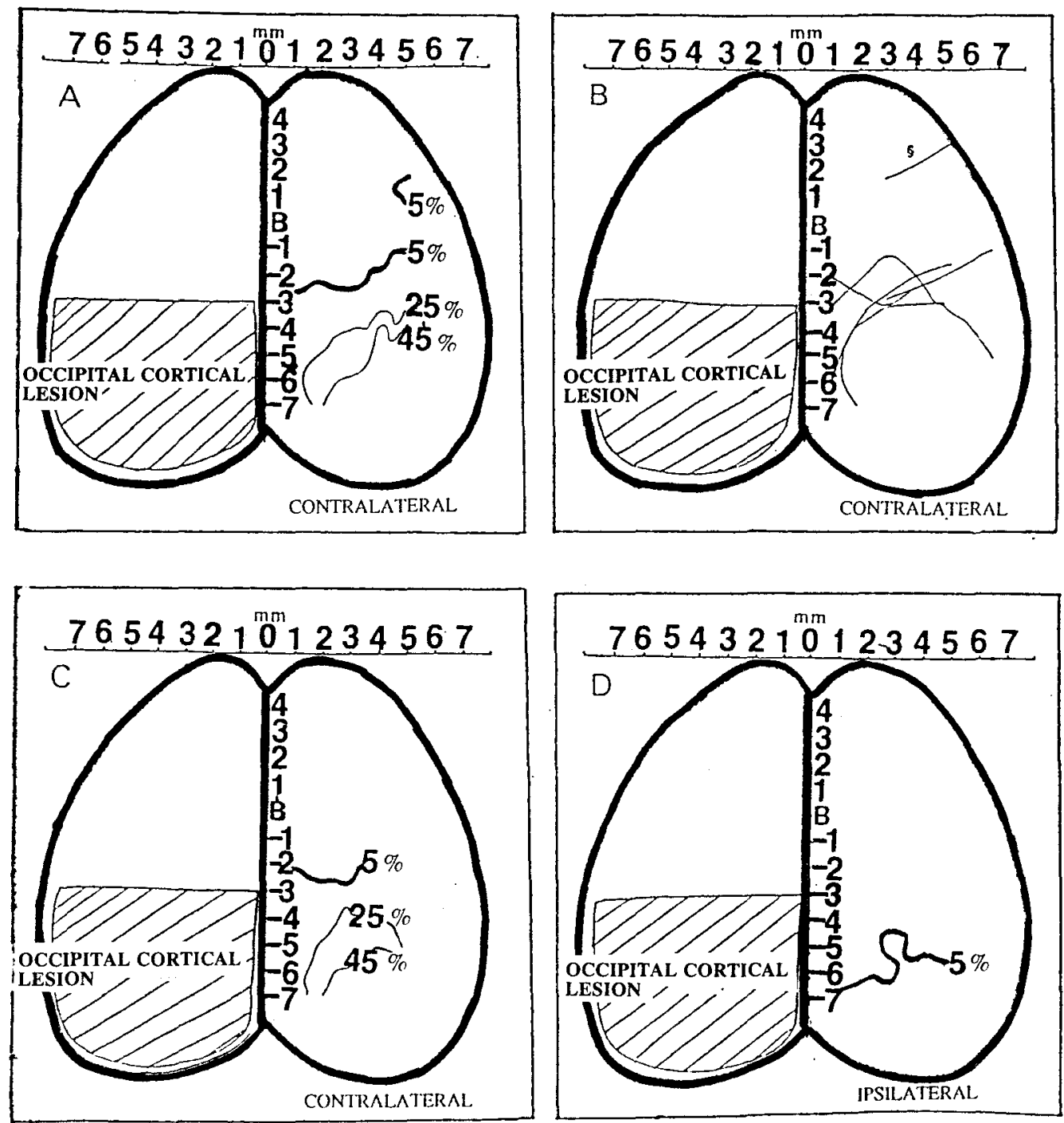
Figure 7. Diagrammatic representations of coronal sections of the $O C$ lesioned brain represented by $\S$ in figure 6, showing the extent and depth of the lesion. The lesion is seen to damage the splenium of the corpus callosum. The sections are taken from the caudal part of the brain and are

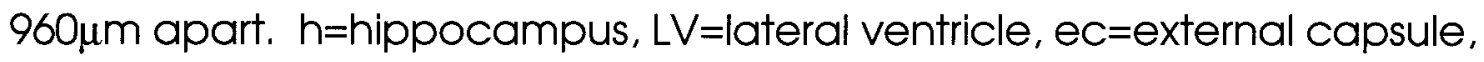
ic=internal capsule, ot=optic tract, $3 \mathrm{~V}=$ third ventricle, $\mathrm{cc}=$ corpus callosum. 


\section{Caudal}

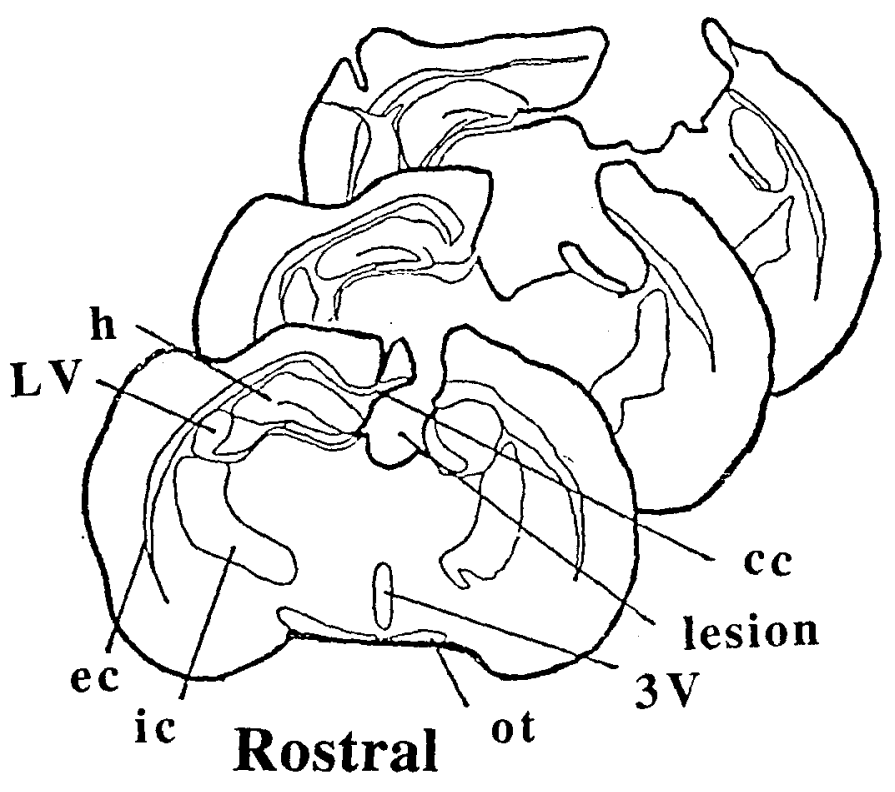


Figure 8. Sagittal sections of a hemisphere from a normal rat ( $a$ and $b$ ) and the unlesioned hemisphere from an SMC rat ( $c$ and $d$ ), stained with toluidine blue and at $3.0 \mathrm{~mm}$ lateral to the midline. (b) and (c) are higher powers of ( $a$ ) and (c) respectively at the border between the visual and sensory cortices to show the point at which the dense fourth layer of the hindlimb sensory area starts. The electrophysiological data obtained at this level are also shown for comparison with the histology. The left of the figure is caudal and the right rostral. $h=$ hindlimb sensory cortex, $f=$ forelimb sensory cortex, OclM=occipital cortex, area 1, Oc2MM=occipital cortex, area 2 , $\mathrm{Br}=$ bregma, $\mathrm{V} 12.2=$ visual evoked potential recorded with an amplitude $12.2 \%$ that of the maximum response recorded using contralateral eye stimulation, $\mathrm{HL}=$ hindlimb motor response with microstimulation, $\mathrm{NR}=\mathrm{no}$ response obtained with microstimulation. The scale bar in (a) and $(c)$ is $1 \mathrm{~mm}$ and in (b) and (d) $200 \mu \mathrm{m}$. 

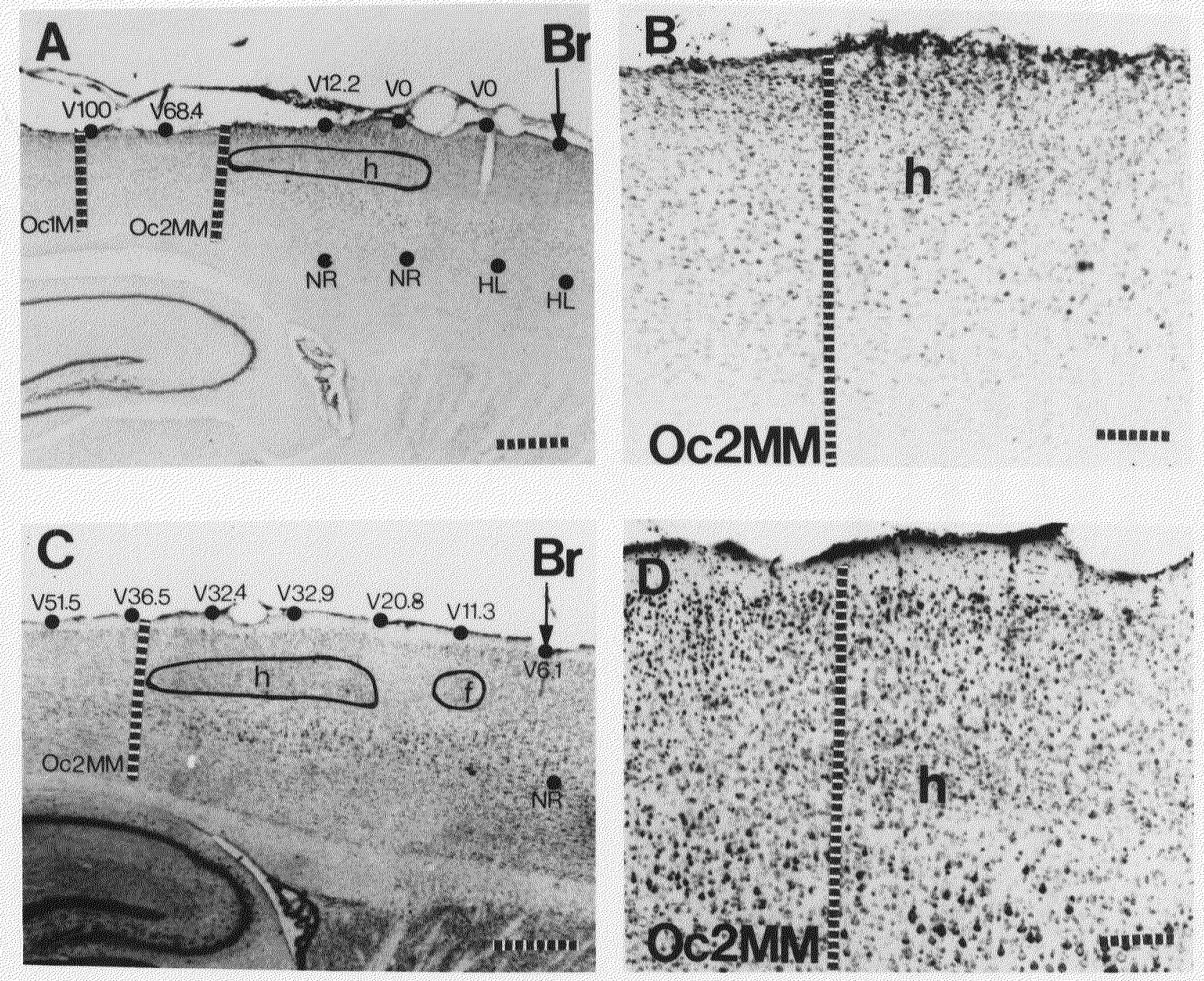
Figure 9. Diagramatic representations of coronal sections of a rat brain taken either at the level of the sensorimotor or visual cortex, and showing a corpus callosal fiber running either from the ablated to the unablated hemisphere ( $a$ ), or from the unablated to the ablated hemisphere (b). The hatched areas represent the cortical lesions in each case. Damage to the cell bodies of callosal fibers running from the ablated to the unablated hemisphere would not be different in their effects on the unablated hemisphere as seen in (a), as they both lead to destruction of the callosal fiber. Fibers running from the unablated to the ablated hemisphere would be differentially affected due to the late arriving visual cortical callosal fibers and would explain our results as shown in the third column of the diagram. 


\section{Callosal Fibers}

(a)

Sensorimotor Cortical

Lesion
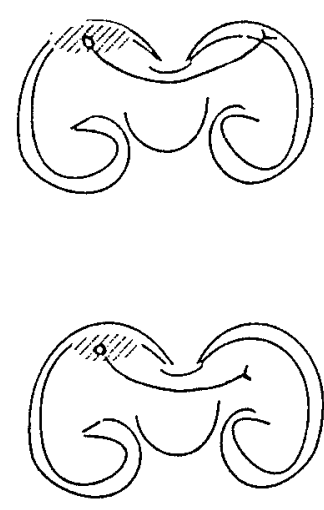

Occipital Cortical

Lesion

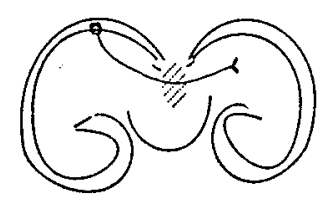

Occipital Cortical Lesion in Posterior Midline

Location (b)
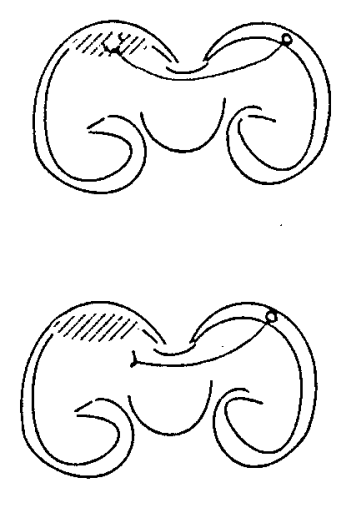

$6(0)$
Change in Visual

Cortical Topography with Lesion

Yes

No

Yes 


\section{Bibliographic Reference Materials}

(1) Alexandrof, Y.I., Grindenko, Y.V. and Jurvelehto, T., Change in the pattern of behavioral specialization of neurons in the motor cortex of the rabbit following lesion of the visual cortex, Acta Physiol. Scand., 139 (1990) 371-385.

(2) Barth, T.M. and Stanfield, B.B., The recovery of forelimb-placing behavior in rats with neonatal unilateral cortical damage involves the remaining hemisphere, J. Neurosci., 10 (1990) 3449-3459.

(3) Boyes, W.K. and Dyer, R.S., Pattern reversal visual evoked potentials in awake rats, Brain Res., 10 (1983) 817-823.

(4) Bronchti, G., Schönenberger, N., Welker, E. and van der Loos, H., Barrelfield expansion after neonatal eye removal in mice, Neurorep., 3 (1992) 489-492.

(5) Castro, A.J., Ipsilateral corticospinal projection after large lesions of the cerebral hemisphere in neonatal rats, Exp. Neurol., 46 (1975) 1-8.

(6) Castro, A.J., Limb preference after lesions of the cerebral hemisphere in adult and neonatal rats, Physiol, \& Behav., 18 (1977) 605-608.

(7) Castro, A.J., Analysis of corticospinal and rubrospinal projections after neonatal pyramidotomy in rats, Brain Res., 144 (1978) 155-158.

(8) Castro, A.J. and Mihailoff, G.A., Corticopontine remodeling after cortical and/or cerebellar lesions in newborn rats, J. Comp. Neurol. 219 (1983) 112-123.

(9) Clark, G., Neurological staining methods. In G. Clark (Ed.), Staining procedures, fourth edition, Williams \& Wilkins, 1981, pp. 142-144.

(10) Cunningham, T.J., Early eye removal produces excessive bilateral branching in the rat: application of cobalt filling method, Science, 194 (1976) 857-858.

(11) Darian-Smith, C. and Gilbert, C.D., Axonal sprouting accompanies functional reorganization in adult cat striate cortex, Nature 368 (1994) 737-740. 
(12) Donoghue, J.P., Suner, S. and Sanes, J.N., Dynamic organization of primary motor cortex output to target muscles in adult rats. II. Rapid reorganization following motor nerve lesions, Exp. Brain Res., 79 (1990) 492-503.

(13) Dyer, R.S., Carolyn, C.C. and Boyes, W.K., Surface distribution of flashevoked and pattern reversal-evoked potentials in hooded rats, Brain Research Bulletin, 18 (1987) 227-234.

(14) Hicks, S.P. and D'Amato, C.J., Motor-sensory and visual behavior after hemispherectomy in newborn and mature rats, Exp. Neurol., 29 (1970) 416-438.

(15) IVy, G.O. Akers, R.M. and Killackey, H.P., Differential distribution of callosal projection neurons in the neonatal and adult rat, Brain Res., 173 (1979) 532-537.

(16) Jacobs, K.M. and Donoghue, J.P., Reshaping the cortical map by unmasking latent intracortical connections, Science, 251 (1990) 944-947.

(17) Jacobson, S. and Trojanowski, J.Q., The appearance of dendrites of callosal and corticothalamic neurons in somatosensory cortex of immature rats demonstrated by horseradish peroxidase. Adv. Neurol., 12 (1975) 319-333.

(18) Jacobson, S., Rich J. and Tovsky, N.J., Delayed myelination and lamination in the cerebral cortex of the albino rat as a result of the fetal alcohol syndrome, Curr. Alcohol., 5 (1979) 123-133.

(19) Jen L.S. and Lund, R.D., Experimentally induced enlargement of the uncrossed retinotectal pathway in rats, Brain Res. 211 (1980) 37-57.

(20) Kaas, J.H. , Merzenich, M.M. and Killackey, H.P., The reorganization of somatosensory cortex following peripheral nerve damage in adult and developing mammals, Ann. Rev. Neurosci., 6 (1983) 325-356.

(21) Kartje-Tillotson, G., Neafsey, E.J. and Castro, A.J., Electophysiological analysis of motor cortical plasticity after cortical lesions in newborn rats, Brain Res., 332 (1985) 103-111.

(22) Kartje-Tillotson, G., Neafsey, E.J. and Castro, A.J., Topography of corticopontine remodeling after cortical lesions in newborn rats, J. Comp. Neurol., 250 (1986) 206-214.

(23) Kartje-Tillotson, G., O'Donoghue, D.L, Dauzvardis, M.F. and Castro, A.J., Pyramidotomy abolishes the abnormal movements evoked by intracortical microstimulation in adult rats that sustained neonatal cortical lesions, Brain Res., 415 (1987) 172-177. 
(24) Killackey, H.P., Chiaia, N.L., Bennett-Clark, C.A., Eck, M. and Rhoades, R.W., Peripheral influences on the size and organization of somatotopic representations in the fetal rat cortex, J. Neurosci., 14 (1994) 1496-1506.

(25) Klüver, H. and Barrera, E. A method for the combined staining of cells and fibers in the nervous system, J. Neuropathol. Exp. Neurol., 12 (1953) 400-403.

(26) Kolb, B. and Gibb. R., Possible anatomical basis of recovery of function after neonatal frontal lesions in rats, Behav. Neuroscl., 107 (1993) 799-811.

(27) Kolb, B. and Whishaw, I.Q., Neonatal frontal lesions in the rat: sparing of learned but not species-typical behaviour in the absence of reduced brain weight and cortical thickness, $J$. Comp. Physiol. Pyschol., 95 (1981) 863-879.

(28) Laemle, L.K. and Labriola, A.R., Retinocollicular projections in the neonatal rat: an anatomical basis for plasticity, Dev. Brain Res., (1982) 317-322.

(29) Land, P.W. and Lund, R.D., Development of the rat's uncrossed retinotectal pathway and its relation to plasticity studies, Science, 205 (1979) 698-700.

(30) Leong, S.K. and Lund, R.D., Anomalous bilateral corticofugal pathways in albino rats after neonatal lesions, Brain Res., 62 (1973) 218221.

(31) Lund, J.P., Sun, G. and Lamarre, Y., Cortical reorganization and deafferentation in adult macaques, Science, 265 (1994) 546-547.

(32) Merzenich, M.M., Kaas, J.H., Wall, J., Nelson, R.J., Sur, H. and Felleman, D., Topographic reorganization of somatosensory cortical areas $3 \mathrm{~B}$ and 1 in adult monkeys following restricted deafferentation, Neurosci., 8 (1983) 33-55.

(33) Miller, M.W. and Vogt, B.A., Direct connection of rat visual cortex with sensory, motor and association cortices, J. Comp. Neurol., 226 (1984) 184-202.

(34) Mooney, R.D., Klein, B.G. and Rhoades, R.W., Effects of altered visual input upon the development of the visual and somatosensory representations in the hamster's superior colliculus, Neuroscience, 20 (1987) 537-555.

(35) Nah, S.H., Ong, L.S. and Leong, S.K., Is sprouting the result of a persistent neonatal connection?, Neurosci. Lett., 19 (1980) 39-44. 
(36) Neafsey, E.J., Bold, E.L., Haas, G., Hurley-Gius, K.M., Quirk, G., Sievert, C.F. and Terreberry, R.R., The organization of the rat motor cortex: a microstimulation mapping study, Brain Res. Rev., 11 (1986) 7796.

(37) Olavarria, J. and Van Sluyters, R., Organization and postnatal development of callosal connections in the visual cortex of the rat, $J$. Comp. Neurol., 239 (1985) 1-26.

(38) Ono, K., Shimada, M. and Yamano, T., Reorganization of the corticospinal tract following neonatal unilateral cortical ablation in rats, Brain and Dev., 12 (1990) 226-236.

(39) Onofrj, M., Harnois, C. and Bodis-Wollner, I., The hemispheric distribution of the transient rat VEP: a comparison of flash and pattern stimulation, Exp. Brain Res., 59 (1985) 427-433.

(40) Perry, V.H. and Cowey, A., A sensitive period for ganglion cell degeneration and the formation of aberrant retinofugal connections following tectal lesions in rats, Neuroscience, 7 (1982) 583-594.

(41) Pons, T.P., Garraghty, P.E., Ommaya, A.K., Taub, E. and Mishkin, M., Massive cortical reorganization after sensory deafferentation in adult macaques, Science, 252 (1991) 1857-1860.

(42) Rauschecker, J.P., Compensatory plasticity and sensory substitution in the cerebral cortex, TINS 18 (1995) 36-43.

(43) Reinoso, B.S. and Castro, A.J., A study of corticospinal remodeling using retrograde fluorescent tracers in rats, Exp. Brain Res., 74 (1989) 387394.

(44) Roe, A.W., Pallas, S.L., Kwon, Y. H. and Sur, M., Visual projections routed to the auditory pathway in ferrets: receptive fields of visual neurons in primary auditory cortex, J. Neurosci., 12 (1992) 3651-3664.

(45) Rouiller, E.M., Liang, F., Moret, V. and Wiesendanger, M. , Trajectory of redirected corticospinal axons after unilateral lesion of the sensorimotor cortex in neonatal rat: a phaseolus vulgaris-leucoagglutin (PHA-L) tracing study, Exp. Neurol., 114 (1991) 53-65.

(46) Ryogo, D.K., Ryogo, R., Globus, A. and Killackey, H.P., Increased spine density in auditory cortex following visual or somatic deafferentation, Brain Res., 90 (1975) 143-146.

(47) Schreyer, D.J. and Jones, E.G., Growth and target finding by axons of the corticospinal tract in prenatal and postnatal rats, Neuroscience, 7 (1982) 1837-1853. 
(48) Senft, S.L. and Woolsey, T.A., Growth of thalamic afferents into mouse barrel cortex, Cerebral Cortex, 1 (1991) 308-335.

(49) Sharp, F.R. and Gonzalez, M.F., Adult rat motor cortex connections to thalamus following frontal cortical lesions: WGA-HRP and amino acid studies, Brain Res., 395 (1986) 169-187.

(50) Shaw, C.A., Lanius, R.A. and van den Doel, K., The origin of synaptic neuroplasticity: crucial molecules or a dynamical cascade? Brain Res, Rev., 19 (1994) 241-263.

(51) Tillotson, G.L., Hogan, T.P., Prince, M.M., Neafsey, E.J. and Castro, A.J., Motor cortical plasticity and the effects of fetal neocortical transplants in rats that sustained cortical lesions at birth, Neurosci. Abstr., 19 (1993).

(52) Toldi, J., Joo, F., Feher, O. and Wolff, J.R., Modified distribution patterns of responses in rat visual cortex induced by monocular enucleation. Neurosci. 24 (1988) 59-66.

(53) Toldi, J., Farkas, T. and Volgyi, B., Neonatal enucleation induces crossmodal changes in the barrel cortex of rat. A behavioral and electrophysiological study, Neurosci .Lett., 167 (1994) 1-4.

(54) Wall, J.T., Huerta, M.F. and Kaas, J.H., Changes in the cortical map of the hand following postnatal median nerve injury in monkeys: modification of somatosensory aggregrates, J. Neurosci., 12 (1992) 3445-3455.

(55) Wall, J.T., Huerta, M.F. and Kaas, J.H., Changes in the cortical map of the hand following postnatal ulna and radial nerve injury in monkeys: organization and modification of nerve dominance aggregrates, $\mathrm{J}$. Neurosci., 12 (1992) 3456-3465.

(56) Wise, S.P. and Jones, E.G., The organization and postnatal development of the commissural projection of the rat somatic sensory cortex, J. Comp. Neurol., 168 (1976) 313-344.

(57) Weiss, D.S. and Keller, A., Specific patterns of intrinsic connections between representation zones in the rat motor cortex, Cereb. Cort., 4 (1995) 205-214.

(58) Yu, X.H., Moret, V. and Rouiller, E.M., Re-examination of the plasticity of the corticothalamic projection after unilateral neonatal lesion of the sensorimotor cortex in the rat: a phaseolus vulgaris-leucoagglutinin tracing study, Journal fur Hirnforschung, 36 (1995) 123-133. 
(59) Zecevic, N., Mojsolovic, J., Novakovic, B. and Rakic, L., Brain plasticity after corpus callosum transection in the newborn rat, Metabolic Brain Disease, 4 (1989) 25-31. 


\section{VITA}

The author, Eleftherios Stelios Papathanasiou, was born on December 21, 1968 in London, England to Stelios and Theognosia Papathanasiou. He recieved his secondary education at the American Academy, Larnaca, Cyprus, graduating in 1987. He earned the Mendelian Prize for the best work in Biology in 1985 and prizes for the best work in Biology, Chemistry and Computers in 1987. After military service he went to King's College, University of London where he earned an Upper Second Class Honours Degree in Blochemistry in August 1991. He then returned to Cyprus where he started a position as a clinical neurophysiologist at the Cyprus Institute of Neurology \& Genetics in the capital Nicosia, performing neurodiagnostic tests such as Electroencephalograms, Evoked Potentials and assisting in Electromyography. He was awarded a Fullbright Scholarship in February 1994 to attend a Masters Program in Neurobiology in the United States of America with the purpose of returning to the Institute after his studies and undertaking the responsibilities of Supervisor of Clinical Neurophysiology at the Institute.

In August 1994, Eleftherios entered the Department of Cell Biology, Neurobiology \& Anatomy of the GraduateSchool at Loyola University Chicago. He joined the laboratory of Dr. Gwendolyn Tillotson to pursue research in visual cortical plasticity after neonatal brain injuries. He was the recipient of a Loyola University Basic Science Fellowship from 1994-1995. 


\section{THESIS APPROVAL SHEET}

The thesis submitted by Eleftherios Stelios Papathanasiou has been read and approved by the following committee:

Gwendolyn L. Tillotson, M.D., PhD., Director

Clinical Assistant Professor, Neurology

Hines VA Hospital \& Loyola University Chicago

Assistant Professor, Cell Biology, Neurobiology \& Anatomy

Loyola University Chicago

Edward J. Neafsey, PhD.

Professor, Cell Biology, Neurobiology \& Anatomy

Loyola University Chicago

Director, Interdepartmental Neuroscience Graduate Program

Loyola University Chicago

Anthony J. Castro, PhD.

Professor, Cell Biology, Neurobiology \& Anatomy

Loyola University Chicago

Neal S. Peachey, PhD.

Assistant Professor, Neurology

Hines VA Hospital \& Loyola University Chicago

The final copies have been examined by the director of the thesis and the signature which appears below verifies the fact that any necessary changes have been incorporated and that the thesis is now given final approval by the committee with reference to content and form.

The thesis is, therefore, accepted in partial fulfillment of the requirements for the degree of Master of Science.

$11 / 30 / 45$

Date

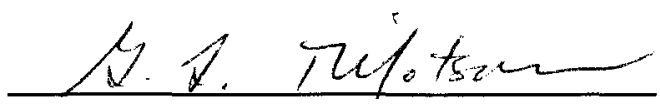

Director's Signature 